\title{
Embedded system based data acquisition and control system for photoacoustic spectroscopic applications
}

\author{
David Tatrai $^{\mathrm{a}, \mathrm{b}, *}$, Zoltan Bozoki ${ }^{\mathrm{a}, \mathrm{b}}$, Gabor Gulyas ${ }^{\mathrm{c}}$, Gabor Szabo ${ }^{\mathrm{a}, \mathrm{b}}$ \\ ${ }^{a}$ University of Szeged, Department of Optics and Quantum Electronics, 6720 Szeged, Dóm tér 9., Hungary \\ ${ }^{\mathrm{b}}$ Hungarian Academy of Sciences, University of Szeged Research Group on Photoacoustic Spectroscopy, 6720 Szeged, Dóm tér 9., Hungary \\ ${ }^{\mathrm{c}}$ Hilase Kft, 6727 Szeged, Irinyi J. utca 1.4/400., Hungary
}

\section{A R T I C L E I N F O}

\section{Article history:}

Received 15 September 2014

Received in revised form 29 November 2014

Accepted 11 December 2014

Available online 23 December 2014

\section{Keywords:}

Photoacoustic spectroscopy

FPGA

Embedded systems

LabVIEW

\begin{abstract}
A B S T R A C T
Laser absorption spectroscopy by photoacoustic (PA) detection technique is increasingly used in trace gas analysis. Our research group focuses on related instrument and application development. In this paper the design and test results of a newly developed field programmable gate array based data acquisition and control system (DACS), are introduced. It became necessary to develop this new system since the limits of the old one had been reached; furthermore, it was challenging to implement new advanced measuring protocols. The system was designed to be scalable in order to be able to implement further ideas or measurement protocols without significant hardware or software modifications. In the current configuration, approximately $60 \%$ of the resources of the system are used. A complete PA measuring system was built around the new DACS to determine the analytical properties: the normalized noise equivalent absorption is $7.5 \times 10^{-10} \mathrm{~cm}^{-1} \mathrm{~W} \mathrm{~Hz}^{-1 / 2}$, while the estimated dynamic range for measuring optical absorption is 7.5 orders of magnitude.
\end{abstract}

(c) 2014 Elsevier Ltd. All rights reserved.

\section{Introduction}

\subsection{Motivation}

In recent years the number of reported photoacoustic (PA) instruments to prove their applicability in various

Abbreviations: AC, Alternating Current; $\mathrm{CH}$, Channel; DACS, Data acquisition and control system; DC, Direct current; FPGA, Field Programmable Gate Array; IC, Integrated Circuit; IO, Input-output; PA, Photoacoustic; PCB, Printed Circuit Board; PI, Proportional-integral; PID, Proportional-integral-derivative; PLL, Phase locked loop; PWM, Pulsewidth modulation; RT, Real Time; SPI, Serial peripheral interface; TDL, Tunable diode laser.

* Corresponding author at: University of Szeged, Department of Optics and Quantum Electronics, 6720 Szeged, Dóm tér 9, Hungary. Tel.: +36 202263707.

E-mail addresses: tatraid@titan.physx.u-szeged.hu (D. Tatrai),zbozoki@ physx.u-szeged.hu (Z. Bozoki),gabor.hulyas@hilase.hu(G. Gulyas),gszabo@ physx.u-szeged.hu (G. Szabo). areas has been steadily increasing [1-4]. Amongst these instruments are those which were developed by our research group for various applications, including in the natural gas industry [5-7], airborne hygrometry [8-9], source apportionment of urban light-absorbing aerosols [10-11], measurements of gas permeability [12], and clinical research [13]. The main components of our instruments are basically always the same: a data acquisition and control system (DACS), one or more longitudinal differential detection cells [14], (a) modulated light source(s) which is/are either telecommunication-type diode lasers [15] or frequency converted Nd:YAG lasers [10] and gas handling units in various configurations.

The DACS has a fundamental role in a PA system. It drives the laser: tunes and stabilizes its wavelength and modulates its emitted light. It amplifies the microphone signal(s) and calculates the PA signal (which is usually defined as the amplitude of the microphone signal at the 
laser modulation frequency). It also calculates either the optical absorption coefficient or the concentration of light-absorbing components from the PA signal based on pre-programmed calibration factors. In most cases, the DACS has various additional functions such as controlling the gas handling system and communicating with other instruments. Our recently developed systems [5-13] were based on a highly integrated target DACS. This system turned out to be one of the bottlenecks during the application developments due to its limited capabilities; therefore, a fundamental redesign of our DACS became essential.

It was decided that the new DACS would be based on a general purpose industry standard, commercial offthe-shelf development platform, which has to be supplemented with additional units only for those functions, which cannot be performed by the platform itself. The new system, its development, and the most important test results are presented in this paper. This paper is organized as follows: First, the salient points about PA spectroscopy are introduced in Section 1.2, focusing on the hardware and software necessities. The materials and methods section (Section 2.) contains the descriptions of the new DACS (hardware and software), the PA system into which it was implemented, and experiments aimed at verifying the performance of the new DACS. In the Results and Discussion section (Section 3.), the most important test results, such as dynamic range and detection limits, are introduced and discussed. In Appendix A, the supplementary homemade peripherals are introduced.

\subsection{Basics of PA spectroscopy}

\subsubsection{The PA effect}

The PA effect is the conversion of electromagnetic radiation to acoustic waves through modulated absorption, non-radiative molecular relaxation, local heating, thermal expansion, and sound wave generation [16-18]. If a gas sample is illuminated by a train of light pulses or by intensity or wavelength-modulated light, which are at least partially absorbed by the sample, the absorbed light energy generates acoustic waves. In most of the PA experimental arrangements, the gas sample being analyzed is introduced into an acoustic resonator [14], which amplifies the acoustic signal (AS) that has been generated, which in turn is measured by a sensitive microphone or by a quartz tuning fork [19]. The amplitude of the signal generated is usually determined by lock-in technique. The amplitude thus determined is usually called as PA signal and will be referred to as such in the followings. In most cases, the PA signal is proportional to the power of the applied light beam, the molar absorption coefficient, and the partial pressure of the absorbing molecules. One multiplicative parameter describes the measurement cell, and another defines the sensitivity of the microphones [20].

$P A=M C P \propto C$

where $M$ is the sensitivity of the microphone, $C$ is the socalled cell constant, $P$ is the power of the light beam, $\alpha$ is the molar absorption coefficient, and $c$ is the concentration of the absorbing molecules.

\subsubsection{Tunable diode laser (TDL)-based PA spectroscopy}

In selective and compact PA instruments nowadays, mostly TDL-s are used as light sources especially the narrow linewidth fiber coupled distributed feedback (DFB) [15] types. DFB diode lasers can be wavelength-tuned by their temperature and by the applied current. Above the operational threshold current, the wavelength and the power of the lasers as the function of temperature and current can be given as:

$$
\begin{aligned}
& \lambda(I, T)=\lambda_{0}+a T+b I \\
& P_{\text {laser }}(I)=P_{0}+f I
\end{aligned}
$$

where $\lambda_{0}$ is a virtual wavelength for $0 \mathrm{~K}$ temperature and $0 \mathrm{~mA}$ current, $a$ is the temperature tuning coefficient (typical value: $100 \mathrm{pm} / \mathrm{K}), T$ is the temperature in $K, b$ is the current tuning coefficient (typical value: $5 \mathrm{pm} / \mathrm{mA}$ ), $I$ is the applied laser diode current; $P_{0}$ is the negative virtual power at $0 \mathrm{~mA}$ current, and $f$ is the slope of the powercurrent curve above the threshold limit (typical value: $0.10-0.30 \mathrm{~mW} / \mathrm{mA}$ ).

The temperature of these lasers is used to stabilize their wavelength, while current is used to fine-tune it [21]. These lasers have an integrated Peltier element and a negative temperature coefficient (NTC) thermistor, which can be used for temperature stabilization through proportional-integral (PI) or proportional-integral-derivative (PID) control methods. The desired stability of the temperature is $0.01 \mathrm{~K}$ or better. For highly accurate and precise temperature and therefore central wavelength stabilization, the temperature of the laser housing also has to be stabilized. For this purpose, an externally placed Peltier element, and an NTC thermistor should be used with PI or PID control.

DFB lasers can be modulated with the modulation of the driving current; such a modulation results in the modulation of both the wavelength and the power. Both of them can generate AS, and their mechanisms have previously been discussed in detail [22-24].

\subsubsection{PA cell}

The PA cell [14] has three major functions: it isolates the gas sample being analyzed, it amplifies the AS that has been generated, and it detects it with the integrated microphones. To do this effectively, the modulation frequency of the laser has to be the same as one of the resonance frequencies of the PA cell itself. During the measurements, a single longitudinal mode differential type was used. The pathlength in such a PA cell is around $10 \mathrm{~cm}$, so the light attenuation is hardly ever more than $10 \%$; therefore, the rest of the light beam can be introduced into a second and maybe third or fourth measuring cell thus making it possible to analyze the same gas multiple times to increase the precision of the measurement by averaging, or to analyze different gas streams simultaneously (Fig. 1).

The resonance frequency of the PA cell depends on its geometry and on the sound velocity within the gas inside. Besides the composition, the sound velocity also depends on the temperature of the gas; therefore, the temperature of the cell has to be stabilized and the sampling line also has to be tempered. These types of PA cells require $0.5 \mathrm{~K}$ 
temperature stability; pulse width modulation PWM heating is usually appropriate for this purpose.

\subsubsection{Optimization}

While optimizing a PAS system, the goal is usually to get as high a signal-to-noise ratio as possible, which is usually equal to as high a signal level as possible at a certain concentration. The goal is to find the optimal laser temperature, modulation depth, and modulation frequency through PA spectrum and resonance curve measurements. The calibration constants also have to be determined by setting different concentrations and measuring the PA signal.

\subsubsection{Accessories}

For proper operation, a PAS system, like any other spectroscopic system designed for gas composition analysis has to be equipped with a gas handling system that consists of flow rate regulation, gas temperature control, and gas pressure stabilization or at least pressure monitoring. The controlling electronics have to be ready to handle them.

\subsection{Requirements for the new DACS}

The new DACS has to fulfill several requirements besides the basic ones, such as being able to be used for PA measurements and having at least as good analytical properties as the previously used system, including autonomous operation and the option of different self-checking procedures:

Advanced measurement techniques: The new DACS has to meet not only current requirements but further needs, such as $2 \mathrm{f}$ detection and simultaneously handling several lasers in the same PA cell.

Ease of testing and programming: Setting a PA system for a new application usually requires intensive optimization of its operational protocol, which can be achieved best if a standard programming language is implemented on the DACS. Since this was not the case with our previous system, this is considered to be one of the fundamental advantages of the upgraded DACS.

Size and weight: Our previously used integrated DACS is $160 \times 250 \times 400 \mathrm{~mm}$ (height $\times$ depth $\times$ width) in size and $7.5 \mathrm{~kg}$ of weight. The new DACS has to be significantly smaller and lighter in order to open up new application possibilities such as operation on unmanned aerial systems.

\section{Materials and methods}

\subsection{The new DACS: Hardware}

The new PA-DACS hardware (Fig. 2) is built out of a National Instrument (NI) SBRIO 9642 controller which is programmable in LabVIEW environment, an NI9234 analogue-digital converter (ADC) module, an NI9269 digital-analogue converter (DAC) module, and various home-made auxiliary units. Practically the same system without significant software modification can be built based on NI CompactRIO systems, which can provide advanced system integration features.

The controller contains a Xilinx Spartan3 field programmable gate array (FPGA) (2 million logic gates, $40 \mathrm{MHz}$ ); a Freescale 5200 Microprocessor (32 bit, $400 \mathrm{MHz}$ ); several integrated analogue and digital input-output (IO) channels $(\mathrm{CH})$; Ethernet and RS232 communication ports; and $256 \mathrm{MB}$ for data storage. The integrated analogue IO CHs are used for purposes requiring less precision measurements, such as pressure sensors (typically $0.5 \mathrm{hPa}$ precision is appropriate), PT100 temperature sensors for $0.05 \mathrm{~K}$ precision, to control flow regulators, and a complete gas handling system. The digital IO CHs are used as PWM sources, for serial peripheral interface (SPI) communication, and Boolean logic controls.

The ADC module has $4 \mathrm{CHs}$ (24 bit resolution, $\pm 5 \mathrm{~V}$ range, $-110 \mathrm{~dB} C H$ to $\mathrm{CH}$ isolation, $51,200 \mathrm{~S} / \mathrm{s} / \mathrm{CH}$ sampling rate) while the DAC module has $4 \mathrm{CHs}$ (16 bit resolution, $\pm 10 \mathrm{~V}$ range; $250 \mathrm{~V}$ RMS $\mathrm{CH}$ to $\mathrm{CH}$ isolation, $100 \mathrm{kS} / \mathrm{s} / \mathrm{CH}$ sampling rate). These modules are used for precisionand timing critical purposes, such as controlling the temperature and the current waveform of the laser and measuring the amplified microphone signals.

\subsubsection{Peripherals}

The designed laser controller, Peltier element driver, and microphone amplifiers are introduced in Appendix A.

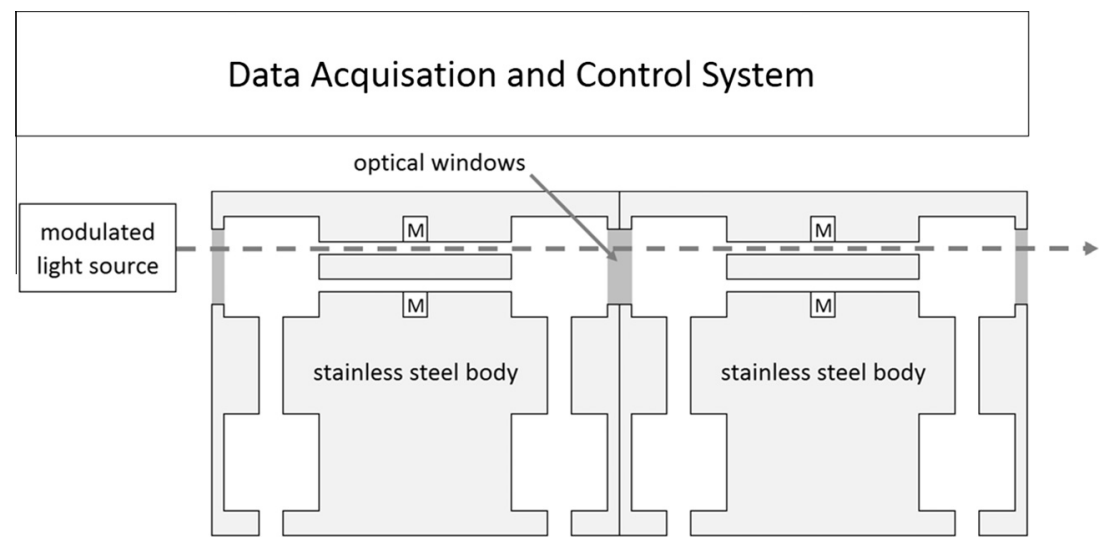

Fig. 1. A basic PA system with differential single longitudinal mode PA cells in dual channel configuration. 


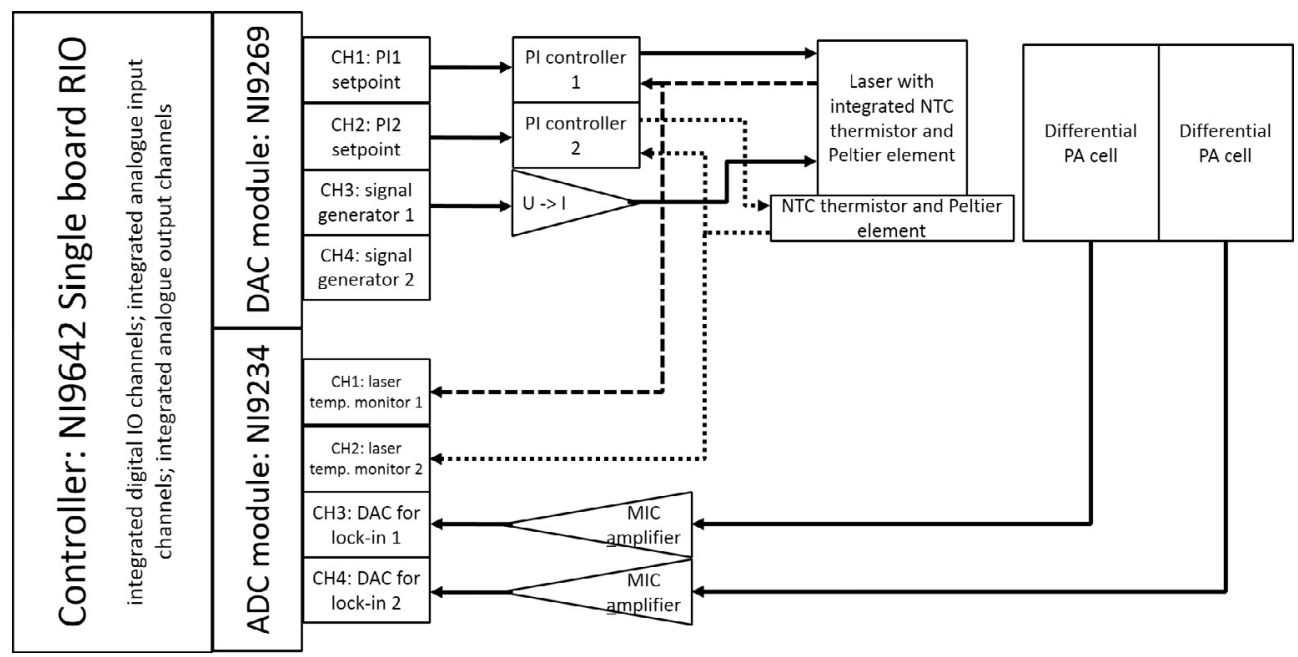

Fig. 2. Schematic layout of the most important functions of the new DACS around a basic PA system. The different types of connecting lines were applied only for better visibility.

Signal conditioning and power circuits had to be designed and constructed for different sensors, such as PT100 temperature sensors, and for PWM-based heating units. These latter components are not critical and so they are not introduced in greater detail.

\subsection{The new DACS: Software}

The self-written software package has three layers: the first is the FPGA code, the second is a Real Time (RT) application that runs on the embedded microprocessor, and the third is an optional Host user interface that runs on an external PC. The FPGA code is responsible for all the timing critical processes and for reading and writing signals from and to each IO $\mathrm{CH}$. The FPGA program also sends and receives commands and messages to and from the RT application. The RT application is responsible for the organization of the measurement loops, for data storage, and for complicated mathematical calculations. The Host program is only a user interface which can be used to send commands to set the desired measurement loop with the proper parameters to the RT application. The Host program can also acquire data that has been measured and evaluated so that it can be saved and visualized on the host PC, and it can be used for any user-desired purposes, such as advanced data processing or integration into a complex measuring system. The RT application can be configured for autonomous operation without human interaction; therefore, in such cases there is no need for the Host program. As the Host program has no effect on the performance of the system it is not introduced or discussed greater in detail. The hierarchy of the different software layers, their connections, and their functions can be seen in Fig. 3.

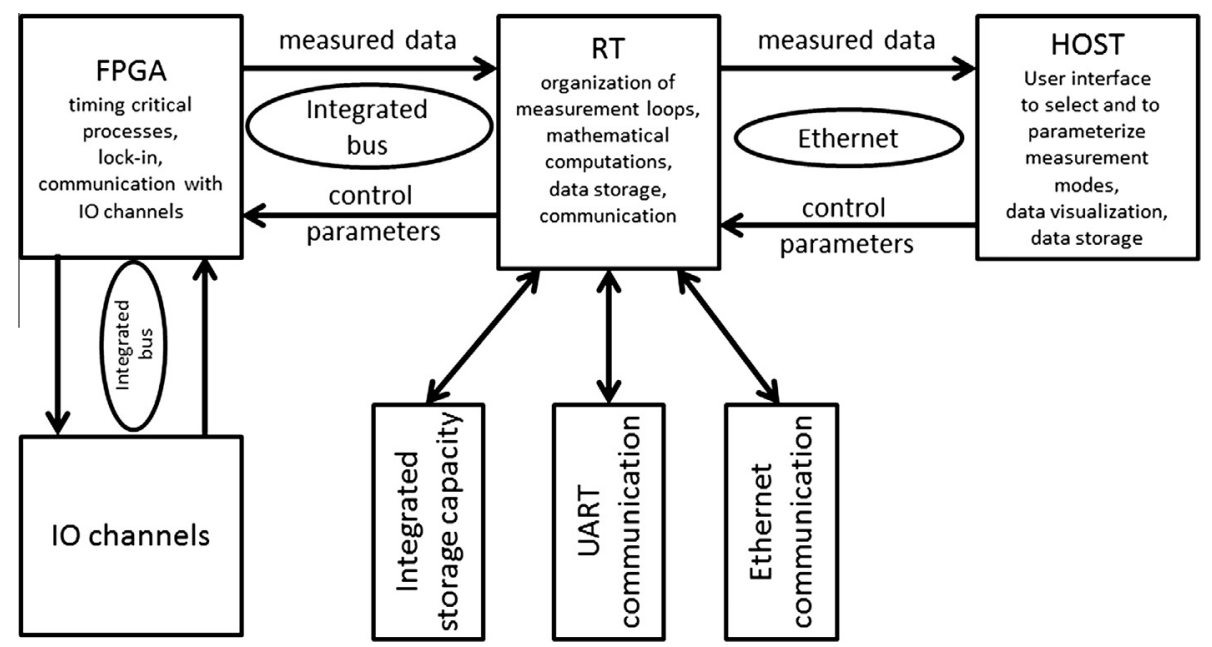

Fig. 3. The block diagram of the functions of the different software layers and the communication between them. Arrows represents the dataflow directions. 


\subsubsection{The FPGA program}

The following main functions are implemented in the FPGA (note: in the following description a one-laser, onecell configuration of the PA system is assumed, but the DACS can be configured for several lasers and/or cells too).

2.2.1.1. Laser temperature stabilization. For each laser driven by the DACS two CHs of the DAC module are programmed to generate direct current (DC) voltage signals to control the PI temperature regulators. One of them is used to stabilize the temperature of the laser chip, and the other to stabilize the temperature of the laser housing. Similarly, for each laser two (DC coupling configured) $\mathrm{CHs}$ of the ADC module are used to monitor the temperature of the laser chip and of the laser housing.

2.2.1.1.1. Performance of the laser temperature regulator. Test measurements, which are not detailed here, proved that the laser temperature can be set with an accuracy of $0.5 \mathrm{mK}$ which is equivalent to a wavelength accuracy of about $50 \mathrm{fm}$. This resolution is sufficient for most PA applications and 20 times better than that of the old DACS.

The standard deviation of the temperature was $0.45 \mathrm{mK}$ during a ten minute period of measurement, while the long term walk-off was less than $1 \mathrm{mK}$ during one day of measurement. The detection limit of these values with the previous DACS was $2.3 \mathrm{mK}$ so the performance of the new system shows a significant improvement.

The repeatability and the hysteresis of the temperature setting were better than $1 \mathrm{mK}$. Whenever the temperature setpoint was modified the temperature of the laser chip was always within $10 \mathrm{mK}$ of target within less than $20 \mathrm{~s}$; in the case of a small, lower than $0.2 \mathrm{~K}$ step within less than $1 \mathrm{~s}$, while the final stabilization sometimes took more than $10 \mathrm{~min}$, the stabilization time was mostly independent of the integration time and was slightly dependent on the proportional gain. The stabilization times should probably be improved in the future.

2.2.1.2. Signal generation for driving the laser. The control voltage signal for each laser is generated by one $\mathrm{CH}$ of the DAC module. The waveform generated is determined by a (DC) level, an alternating current (AC) level, the frequency and the type of modulation signal which can be set as a sine or square wave, while additional waveforms can also be implemented [21,25]. The values of the modulation part of the signal are calculated by a normalized phase-locked-loop (PLL) based signal generator and multiplied with the (AC) level. The DC level is added to the modulation signal and limited to the $0-10 \mathrm{~V}$ range by software. Afterwards the calculated signal is produced by the DAC card at $100 \mathrm{kS} / \mathrm{s}$.

2.2.1.3. Measuring and processing the signals from the microphone amplifier(s). One $\mathrm{CH}$ of the ADC module is programmed to measure the AC coupled signal of the microphone amplifier at a sampling rate of $51,200 \mathrm{~s} / \mathrm{s} / \mathrm{CH}$. These digitized signals are processed by PLL-based digital lock-in technique simultaneously at the modulation frequency and at its selected overtones. Test measurements, which are not detailed here, yielded the following parameters for the digital lock-in amplifier implemented: the lock-in amplifier has a linear performance of over 6.6 orders of magnitude dynamic range from $1 \mu \mathrm{V}$ up to $4.5 \mathrm{~V}$ at $1 \mathrm{~s}$ integration time, which is approximately 150 times bigger than that of the old DACS. The optimal averaging time of the lock-in amplifier is $4700 \mathrm{~s}$ where the noise equivalent signal level is $750 \mathrm{pV}$ which amounts to 9.8 orders of magnitude dynamic range. The drift of the signal generation and of the lock-in amplifier was less than $0.2 \sigma$ through 1.5 months. Furthermore, no significant crosstalk between any measuring $\mathrm{CH}$ s of the lock-in amplifier was observed. The schematic layout of the signal generator and of the lock-in amplifier can be found in Fig. 4.

2.2.1.4. Additional implemented functions. In the following, the other functions implemented are listed without detailed explanation; their function is either trivial or not critical to PA applications.

- 6 differential analogue input CHs.

- 4 CHs for measuring temperature with PT100 sensors through Wien-bridge.

- 20 CHs single ended analogue inputs.

- 4 CHs of analogue output for low response time units, such as mass flow controllers.

- 5 PWM CHs at $3.3 \mathrm{~V}$.

- 5 PWM CHs at $24 \mathrm{~V}$.

- 8 SPI CHs.

\subsubsection{The RT application}

The various measurement protocols implemented are coded as subroutines, which makes it possible to maintain and scale the code. Now these routines can be called in a multiple selection case structure. The RT application can be configured to start measurements autonomously with predefined parameters whenever the system is powered up as well. In this operational mode, it is advantageous to organize the different measurement protocols in state machine architecture, which is easy to implement.

The RT microprocessor in the system can be used to run many of the standard LabVIEW functions, including floating point complex calculations. Thanks to these possibilities, the data evaluation process can be automated more easily than with the previous DACS, which was only capable of integer arithmetic calculations.

2.2.2.1. Implemented measurement protocols. Besides a fully manual control mode when each parameter (laser temperature, modulation frequency AC and DC values of the laser driving signal, and averaging time) can be set manually several others were implemented as subroutines such as optical absorption spectrum measurement mode, modulation optimization mode, and resonance frequency search mode. In each case, the integration time of the PA signal can be set in the $0.01-1000 \mathrm{~s}$ range.

\subsection{The PA system into which the new DACS was implemented}

The PA system with which the developed DACS was tested contained a single longitudinal mode differential PA cell with integrated microphones (Knowles EK 3029). 


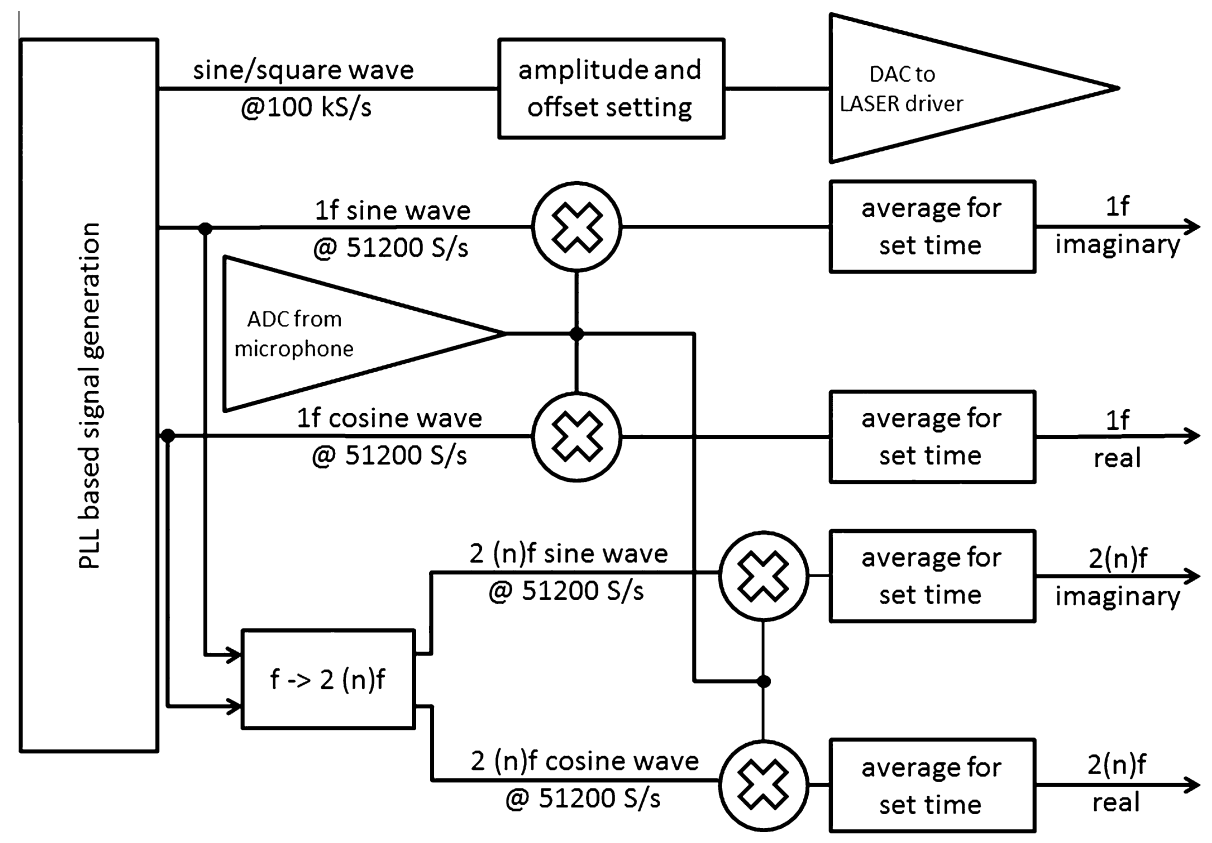

Fig. 4. The schematic logic of the laser modulation signal generation and of the multiply $f$ lock-in signal processing.

The cell was temperature stabilized to $313.15 \pm 0.1 \mathrm{~K}$ $\left(40{ }^{\circ} \mathrm{C}\right)$. A DFB diode laser (NEL NLK1E5GAAA) $(24.6 \mathrm{~mW}$ optical power at $120 \mathrm{~mA}$ DC current) was used as light source, which is tunable in the $1392-1395 \mathrm{~nm}$ range, where water vapor has several strong absorption lines and methane has a weak broadband absorption. The different parts of the gas handling system were connected by $6 \mathrm{~mm}$ stainless steel tubes using Swagelok tube fittings. This PA system is identical to that used in our previous study [8]; only the DACS has been changed, thus making it possible to compare the performance of the two DACSs.

\subsection{Verifying experiments}

\subsubsection{Spectral measurements}

Amplitude and wavelength modulated optical absorption spectrums were measured and compared to a water vapor absorption spectrum database [26]. The $2 \mathrm{f}$ measurement mode was tested during spectrum measurements only to demonstrate the proper operation of the written FPGA code.

\subsubsection{Calibration measurements}

To determine sensitivity the system was calibrated for water vapor from a homemade humidity generator [8] which is capable of generating humidified gas in the $10-25,000$ ppm range. First, calibration occurred on the strongest water vapor absorption line $(1392.5 \mathrm{~nm}$, $3.7 \times 10^{-5} \mathrm{ppm} \mathrm{m}^{-1}$ ) available in the tuning range of the laser with optimized modulation parameters. The evaluation of the calibration revealed that the measurement range of the PA system exceeds the concentration i.e. the absorption coefficient range which can be generated by the generator considerably. Therefore, the calibration was repeated by tuning the laser to a weaker absorption line $\left(1392.8 \mathrm{~nm}, 1.5 \times 10^{-6} \mathrm{ppm} \mathrm{m}^{-1}\right)$ and the light power was simultaneously reduced from $24.6 \mathrm{~mW}$ to $5 \mathrm{~mW}$ by reducing the laser current to $25 \mathrm{~mA}$.

\subsubsection{PA stability test}

Pure methane was flushed through the PA cell from a gas bottle and measurements were performed for one hour with $1 \mathrm{~s}$ averaging time at two different wavelengths, i.e. at different absorption coefficients, where the PA signals were close to the lower limit of detection of the system. The determined statistical parameters and the previously determined sensitivity value were used to determine the lower limit of detection.

\section{Results and discussion}

\subsection{System load}

The FPGA code utilizes 60\% of the Slice Registers, 30\% of the Look Up Tables, $80 \%$ of the Multi $18 \times 18$ blocks and $70 \%$ of the Block RAMs of the FPGA without any timing restriction. The average load of the RT CPU was $18 \%$ and never exceeded 39\%. These load results not only show that the developed DACS is suited to handling present needs but a significant portion of resources is also available for further improvements.

\subsection{Analytical performance of the system}

\subsubsection{Spectral measurements}

The amplitude modulation spectrum (Fig. 5.) was compared to database values and good agreement can be observed. The $1 \mathrm{f}$ and $2 \mathrm{f}$ spectrums measured with low 


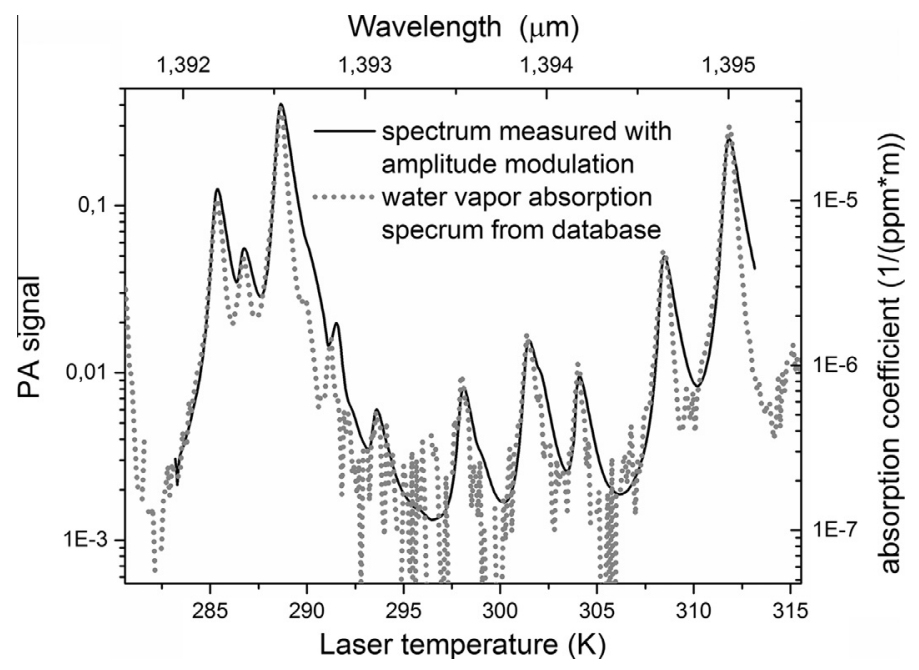

Fig. 5. Comparison of PA spectrum measured with $90 \%$ of modulation depth and of database [26] values.

modulation depth produce the characteristic first and second derivate of the absorption lines (Fig. 6.). These measured spectrums indicate in a qualitative way that the wavelength tuning and laser modulation operate according to preliminary expectations.

\subsubsection{Detection limits}

The results of the calibration at both absorption lines can be seen in Fig. 7. (Here the data which represent measurements on the weaker absorption line and reduced light power are scaled up with the light power ratio.) A linear behavior was observed over 4.5 orders of magnitude variation in the absorption coefficient, while the highest PA signal was approximately 10 times below the upper detection limit of the lock-in itself. This shows that one order of magnitude higher absorption coefficient can still be measured with the same laser without saturation. Therefore the dynamic range of the PA system is around 5.5 orders of magnitude of absorption coefficient and thus of concentration at $1 \mathrm{~s}$ integration time. The sensitivity of the system is $0.64 \mathrm{~V} \mathrm{~m}^{-1}$.

Measurements at constant, low-level PA signals, i.e. when methane was introduced into the cell, resulted in a noise level $(1 \sigma)$ of $6.1 \mu \mathrm{V}$ in the case of $1 \mathrm{~s}$ integration time. From the Allan deviation plot (Fig. 8), the minimum Allan deviation is $2 \times 10^{-7} \mathrm{~V}$ at $270 \mathrm{~s}$ integration time. Therefore, the minimal detectable change of the absorption coefficient, which is defined as $3 \cdot \sigma$ sensitivity divided by analyzer sensitivity, is $2.9 \times 10^{-5} \mathrm{~m}^{-1}$ at $1 \mathrm{~s}$ integration time and $9.4 \times 10^{-7} \mathrm{~m}^{-1}$ at $270 \mathrm{~s}$. Combining these values with the sensitivity and the upper detection limit of the lock-in amplifier, the dynamic range of the system is 5.8 and 7.5 orders of magnitude in the case of 1 and $270 \mathrm{~s}$ integration times, respectively. These values correspond to

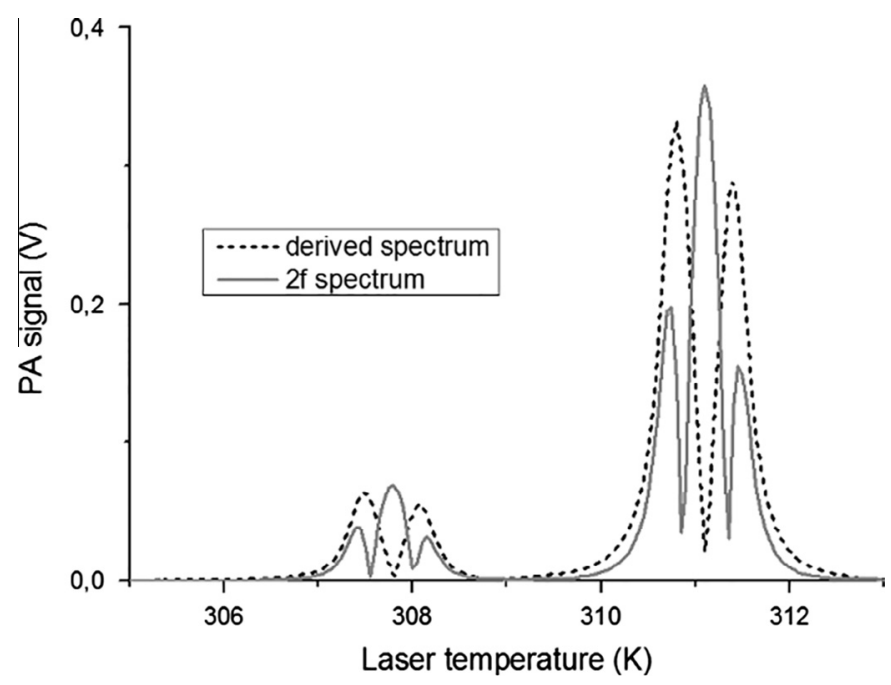

Fig. 6. $1 \mathrm{f}$ and $2 \mathrm{f}$ PA spectrums measured with $15 \%$ modulation depth. The PA signal of the $2 \mathrm{f}$ spectrum was multiplied by 50 for better visibility. 


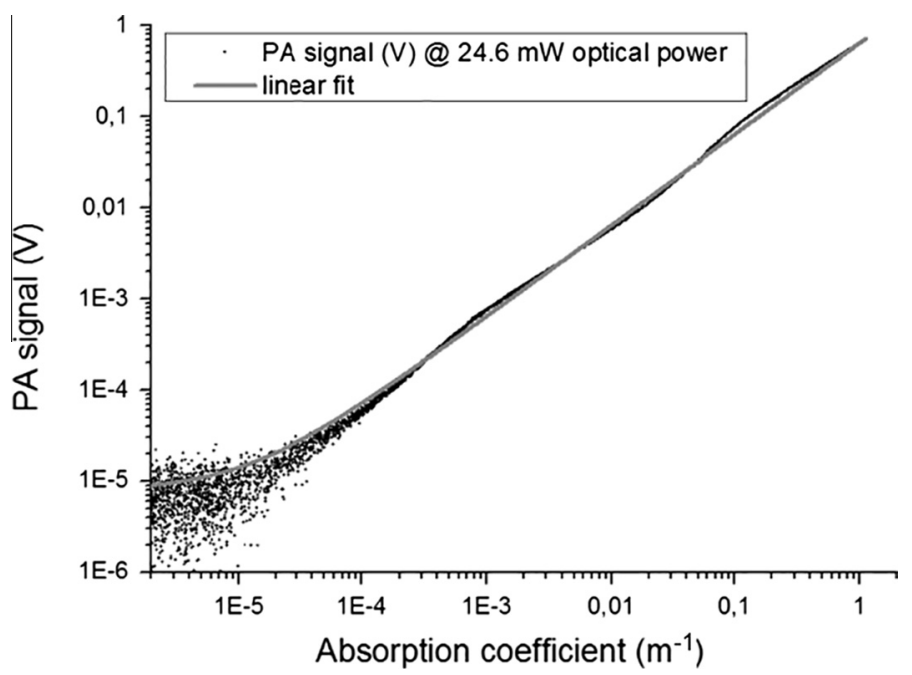

Fig. 7. Calibration curve of PA measurements at both absorption lines. Slope of the fitted calibration line (i.e. sensitivity) is $0.64 \mathrm{~V} / \mathrm{m}^{-1}$.

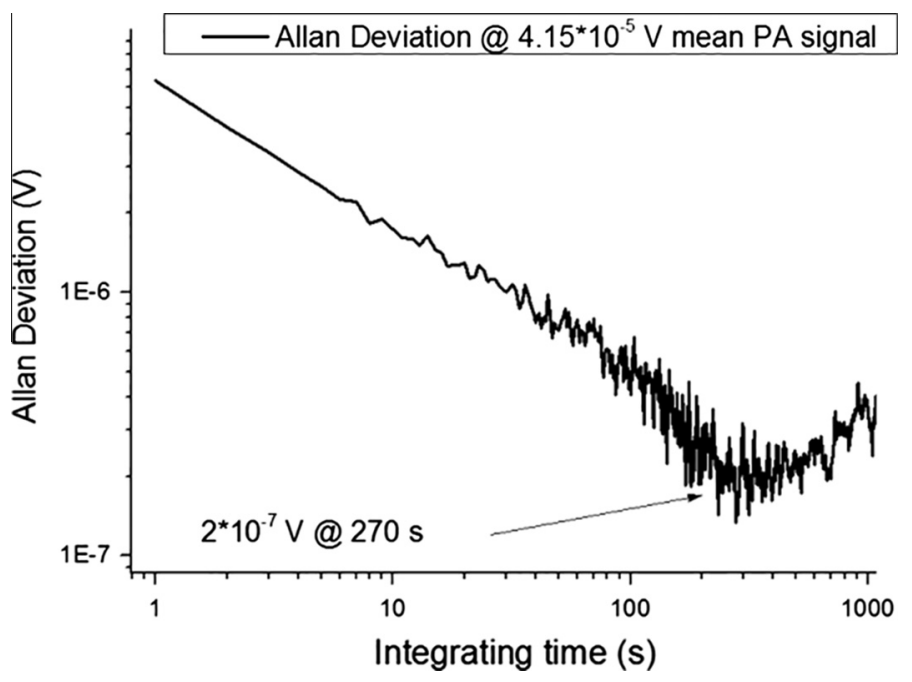

Fig. 8. Allan deviation of PA measurements at low signal values on pure methane. Uncertainty of $210^{-7} \mathrm{~V}$ can be reached with $270 \mathrm{~s}$ averaging time.

$7.5 \times 10^{-10} \mathrm{~cm}^{-1} \mathrm{~W} \mathrm{~Hz}^{-1 / 2}$ normalized noise equivalent absorption, which is comparable to or better than the previously reported values [2].

The old DACS has a dynamic range of 6000 and therefore can only be used in high dynamic range applications several automatic sensitivity modifications are introduced into its operation [8], but the new DACS, thanks to its 5.8 orders of magnitude dynamic range, can easily be operated in such applications without the need to modify its sensitivity.

\subsection{Size and weight}

Though the DACS is not yet assembled inside an instrument rack, its size and weight can already be estimated at only about one fifth of that of the old DACS. The size reduction attained makes it possible at least in the case of simpler PA analyzers for the DACS, the laser, and the PA cells can be assembled inside a standard instrument rack with a width of $19 \mathrm{in}$., a height of 3 units, and a depth of $11 \mathrm{in..}$

\section{Conclusions}

In order to serve as a critical component of new PA measuring instruments, a DACS was developed and successfully tested. The new DACS has improved measurement capabilities, it is easy to use and to configure for further applications, and its size and weight are significantly lower than those of our old DACS. The most important analytical properties have also been improved both with respect to 
sensitivity and dynamic range. These results make it possible for the new DACS to replace our old system with the option of implementing new measurement techniques and of further application developments.

Besides the improved PA measurement capabilities the various functions of the new DACS, such as the highly versatile signal generator and the implemented lock-in amplifier, also make the new DACS attractive for non-PA applications whenever high precision and extremely wide dynamic range are important.

\section{Acknowledgements}

The project was supported by the Hungarian Research and Technology Innovation Fund (OTKA), NN 109679. The research leading to these results has also received funding from the European Community's Seventh Framework Programme (FP7/2007-2013) under grant agreement $n^{\circ}$ 312311.

The authors would like to thank National Instruments Hungary Kft. for providing a CompactRIO system at the beginning of the developments.

\section{Appendix A. The home made peripherals}

\section{A.1. Laser driver}

The laser driver circuit is based on an ATLS250MA103 laser driver integrated circuit (IC) (Analog Technologies) which is a voltage-to-current converter (0-2.5 V-0$250 \mathrm{~mA}$ ) designed for driving diode lasers; its current can be limited with a resistor connected between two pins. The laser used during the tests is a 0-120 mA drivable type; the current limit was set to $118 \pm 1 \mathrm{~mA}$. Any ranges can be set up to $250 \mathrm{~mA}$ with the same laser driver IC by replacing three resistors on the printed circuit board (PCB).

\section{A.2. Laser temperature stabilizer}

The laser temperature stabilization circuit is based on a HTC1500-62 controller (Wavelength Electronics), which is a PI control loop based 1.5 A Peltier-element driver specially designed for diode laser applications. The proportional gain can be set from 1 to 75 with a digital (SPI controlled) potentiometer depending on the actual needs while the integration time is set to $3 \mathrm{~s}$ fixed. The desired temperature can be set by a DC voltage level.

\section{A.3. Microphone amplifier}

The microphone amplifier is based on an AC coupled INA128 (Texas Instruments) instrumentation amplifier. The microphone signals are first band-pass (800$12,000 \mathrm{~Hz}$ ) filtered and then connected to the inputs of the instrumentation amplifier. The output of the INA128 is fed back to its reference point with an OPA177 (Texas Instruments) operational amplifier through a high-pass $(100 \mathrm{~Hz}) \mathrm{RC}$ filter, similarly as recommended on the datasheet. The gain is set to 50 times. With this gain the self-noise of the microphones used is about one order of magnitude higher than the detection limit of the lock-in amplifier. The microphone amplifier was made on a standalone PCB for each measuring cell and mounted on top of the cells to be as close to the microphones as possible.

\section{A.4. Design rules}

During the design of the peripherals, the most important requirement was to minimize the crosstalk between them and to minimize their temperature dependency. To achieve these goals, each unit has its dedicated regulated power source; regulation is done with LM78xx, LM79xx and LM78Sxx voltage regulators and by tantalum capacitors. Furthermore, all components used such as resistors, potentiometers, and filtering capacitors were selected from low thermal coefficient series.

The laser driver and the two PI stabilizers are placed on a common PCB; they are placed and wired at least $5 \mathrm{~mm}$ from each other except at their connection to the laser.

The laser driver IC and PI temperature controller product lines include models with higher output current with the same package and pinout so the same (PCB) can be used to drive both higher current lasers and Peltier cells. The PCB was designed to be able to handle current of up to $1.5 \mathrm{~A}$ for the laser driver and $5 \mathrm{~A}$ for the PI controller.

\section{References}

[1] Z. Bozóki, A. Pogány, G. Szabó, Photoacoustic instruments for practical applications: present, potentials, and future challenges, Appl. Spectrosc. Rev. 46 (2011) 1-37, http://dx.doi.org/10.1080/ 05704928.2010.520178.

[2] P. Patimisco, G. Scamarcio, F.K. Tittel, V. Spagnolo, Quartz-enhanced photoacoustic spectroscopy: a review, Sensors 14 (4) (2014) 61656206, http://dx.doi.org/10.3390/s140406165.

[3] J. Uotila, J. Lehtinen, T. Kuusela, S. Sinisalo, G. Maisons, F. Terzi, I. Tittonen, Drug precursor vapor phase sensing by cantilever enhanced photoacoustic spectroscopy and quantum cascade laser, Proc. SPIE 8545-8 (2012), http://dx.doi.org/10.1117/12.974508.

[4] C.B. Hirschmann, J. Lehtinen, J. Uotila, S. Ojala, R.L. Keiski, Sub-ppb detection of formaldehyde with cantilever enhanced photoacoustic spectroscopy using quantum cascade laser source, Appl. Phys. B 111 (4) (2013) 603-610, http://dx.doi.org/10.1007/s00340-013-5379-4.

[5] A. Varga, Z. Bozoki, M. Szakáll, G. Szabó, Photoacoustic system for online process monitoring of hydrogen sulfide (H2S) concentration in natural gas streams, Appl. Phys. B 85 (2006) 315-321, http:// dx.doi.org/10.1007/s00340-006-2388-6.

[6] H. Huszár, Z. Bozoki, A. Mohacsi, S. Puskas, J. Tamas, G. Szabo, Combination of photoacoustic detector with diffusion sampler for the measurement of water vapor concentration in ethylene glycols for the natural gas industry, Sens. Actuators B 119 (2006) 600-607, http://dx.doi.org/10.1016/j.snb.2006.01.011.

[7] V. Hanyecz, A. Mohácsi, S. Puskas, A. Vago, G. Szabo, Instrument for benzene and toluene emission measurements of glycol regenerators, Meas. Sci. Technol. 24 (2013) 115901, http://dx.doi.org/10.1088/ 0957-0233/24/11/115901.

[8] D. Tatrai, Z. Bozoki, H. Smit, C. Rolf, N. Spelten, M. Krämer, A. Filges, C. Gerbig, G. Gulyas, G. Szabo, Dual channel photoacoustic hygrometer for airborn measurements: background, calibration, laboratory and in-flight inter-comparison tests, Atmos. Meas. Tech. Discuss. 7 (2014) 6359-6384, http://dx.doi.org/10.5194/amtd-7-6359-2014.

[9] M. Szakall, Z. Bozoki, A. Mohacsi, A. Varga, G. Szabó, Diode Laser Based Photoacoustic Water Vapor Detection System for Atmospheric Research, Appl. Spectrosc. 58 (2004) 792-798, http://dx.doi.org/ 10.1366/0003702041389373.

[10] T. Ajtai, A. Filep, M. Schnaiter, C. Linke, M. Vragel, Z. Bozoki, G. Szabo, T. Leisner, A novel multi-wavelength photoacoustic spectrometer for the measurement of the UV-vis-NIR spectral absorption coefficient of atmospheric aerosols, J. Aerosol Sci. 41 (11) (2010) 1020-1029, http://dx.doi.org/10.1016/j.jaerosci.2010.07.008. 
[11] N. Utry, T. Ajtai, A. Filep, M. Pinter, Z.s. Torok, Z. Bozoki, G. Szabo, Correlations between absorption Angström exponent (AAE) of wintertime ambient urban aerosol and its physical and chemical properties, Atmos. Environ. 91 (2014) 52-59, http://dx.doi.org/ 10.1016/j.atmosenv.2014.03.047.

[12] Z. Filus, N. Toth, G. Gulyas, T. Guba, G. Szabo, Z. Bozoki, Carrier gas flow arrangement based photoacoustic detection method for measuring gas permeability of polymer membranes, Polym. Test. 32 (6) (2013) 1099-1104, http://dx.doi.org/10.1016/ j.polymertesting.2013.06.011.

[13] E. Tuboly, A. Szabo, G. Eros, A. Mohacsi, G. Szabo, R. Tengolics, G. Rakhely, M. Boros, Determination of endogenous methane formation by photoacoustic spectroscopy, J. Breath Res. 7 (2013) 046004 , http://dx.doi.org/10.1088/1752-7155/7/4/046004.

[14] A. Miklos, P. Hess, Z. Bozoki, Application of acoustic resonators in photoacoustic trace gas analysis and metrology, Rev. Sci. Instrum. 72 (2001) 1937-1955, http://dx.doi.org/10.1063/1.1353198.

[15] J. Seufert, M. Fischer, J. Koeth, R. Werner, M. Kamp, A. Forchel, DFB laser diodes in the wavelength range from $760 \mathrm{~nm}$ to $2.5 \mu \mathrm{m}$, Spectrochim. Acta Part A: Mol. Biomol. Spectrosc. 60 (2004) 32433247, http://dx.doi.org/10.1016/j.saa.2003.11.043.

[16] A.G. Bell, On the production and reproduction of sound by light: the photophone, Am. J. Sci. 3 (1880) 305-324.

[17] A.G. Bell, The production of sound by radiant energy, Science 2 (1881) 242-253, http://dx.doi.org/10.1126/science.os-2.49.242.

[18] J.F.R.S. Tyndall, Action of an Intermittent beam of radiant heat upon gaseous matter, Science (1881)Vol. os-2 (38) pp. 110-114. http:// dx.doi.org/10.1126/science.os-2.38.110-a.
[19] A.A. Kosterev, Yu.A. Bakhirkin, R.F. Curl, F.K. Tittel, Quartz-enhanced photoacoustic spectroscopy, Opt. Lett. 27 (21) (2002) 1902-1904, http://dx.doi.org/10.1364/OL.27.001902.

[20] F.A. McDonald, G.C. Wetsel Jr., Generalized theory of the photoacoustic effect, J. Appl. Phys. 49 (1978) 2313-2322, http:// dx.doi.org/10.1063/1.325116.

[21] D. Tatrai, Z. Bozoki, G. Szabo, Method for wavelength locking of tunable diode lasers based on photoacoustic spectroscopy, Opt. Eng. 52 (2013) 096104, http://dx.doi.org/10.1117/1.0E.52.9.096104.

[22] S.L. Castleden, G.E. Kirkbright, D.E.M. Spillane, Wavelength modulation in photoacoustic spectroscopy, Anal. Chem. 53 (1981) 2228-2231, http://dx.doi.org/10.1021/ac00237a019.

[23] J. Saarela, J. Toivonen, A. Manninen, T. Sorvajärvi, R. Hernberg, Wavelength modulation waveforms in laser photoacoustic spectroscopy, Appl. Opt. 1 (2009) 743-748, http://dx.doi.org/ 10.1364/AO.48.000743.ez nincs itt.

[24] S. Schilt, L. Thevenaz, Wavelength modulation photoacoustic spectroscopy: Theoretical description and experimental results, Infrared Phys. Technol. 48 (2006) 154-162, http://dx.doi.org/ 10.1016/j.infrared.2005.09.001.

[25] M. Szakall, A. Varga, A. Pogany, Z. Bozoki, G. Szabo, Novel resonance profiling and tracking method for photoacoustic measurements, Appl. Phys. B. 94 (2009) 691-698, http://dx.doi.org/10.1007/s00340009-3391-5.

[26] S.W. Sharpe, T.J. Johnson, R.L. Sams, P.M. Chu, G.C. Rhoderick, P.A Johnson, Gas-phase databases for quantitative infrared spectroscopy, Appl.Spectrosc. 58 (2004) 1452-1461, http:// dx.doi.org/10.1366/0003702042641281. 